\title{
Wenchuan earthquake: response of Chinese dental professionals
}

\author{
J. Dai ${ }^{1}$ Y. Zhao² and G. Li
}

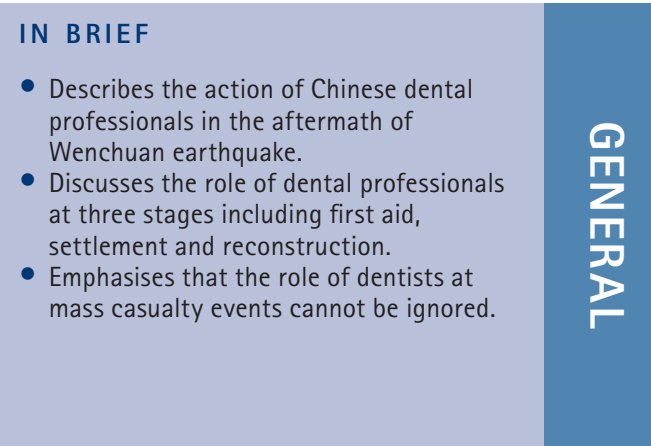

On 12 May 2008, an earthquake with a magnitude of 8.0 on the Richter scale hit Wenchuan, China. In the aftermath of this natural disaster, Chinese dental professionals actively participated in the first emergency medical response team, definitive dental treatment, oral health services and education, and the recovery of local oral care infrastructure and resources. Learning from the experience and first-hand data of the Wenchuan earthquake, dental professionals can increase their awareness of the importance of collaborative emergency response health services in mass casualty events. Further research and emphasis is needed to encourage the participation of dental professionals in disaster preparation training and practice.

\section{INTRODUCTION}

At $2.28 \mathrm{pm}$ on 12 May 2008, a huge earthquake with a magnitude of 8.0 on the Richter scale hit China with its epicentre in Wenchuan (Fig. 1). The Wenchuan earthquake had an extremely high intensity with sustained impact over a large area including the provinces of Sichuan, Shaanxi, Gansu, and the Chongqing Municipal area, in total comprising 417 counties in 16 provinces and municipalities with an area of more than $440,000 \mathrm{~km}^{2}$ - a region so large that close to half of China was affected. More than 15 million buildings collapsed during the earthquake and resulted in direct property damage to buildings and infrastructure of more than US\$150 billion. ${ }^{1}$ According to the statistics data of the Ministry of Civil Affairs of the PRC, up to 16 June 2008 there were 69,170 people confirmed dead, 374,159 seriously injured, and 17,426 reported missing. ${ }^{2}$ All together, the Wenchuan earthquake impacted a population of 46.24

\footnotetext{
$1,3^{*}$ Department of Preventive Dentistry, ${ }^{2}$ Department of Prosthodontics, School of Stomatology, Fourth Military Medical University, Xi'an, Shaanxi China, 710032

${ }^{*}$ Correspondence to: Professor Gang Li

Email: ligangi@fmmu.edu.cn
}

\section{Refereed Paper}

Accepted 11 February 2009

DOI: $10.1038 /$ sj.bdj.2009.167

${ }^{\circledR}$ British Dental Journal 2009; 206: 273-276 million, which was the worst to strike China since the catastrophic Tangshan earthquake in 1976.

Immediately after the quake, with saving lives the first priority, the Chinese government began real-time video/ audio broadcasting. The response of the Chinese people was worthy of the size of the event and relief from various sources was well coordinated. Chinese Prime Minister Wen Jiabao flew to the epicentre of the earthquake and personally oversaw relief work. Chinese troops were mobilised to the affected areas, no matter how remote, to save every life possible from under the rubble. Emergency aid was air-dropped into areas that had been cut off by the disaster. Organised units, teams and experts took prompt action to rescue victims of the earthquake and volunteers from all corners of China travelled to the affected region to offer help, or sent financial aid to the victims. Furthermore, the wise decision was made to accept assistance from international professional medical rescue teams. Many miracles took place which smashed several previous records in the history of earthquake medical rescue.

Most importantly, the Chinese Ministry of Health's timely implementation of emergency earthquake procedures meant that designated medical emergency teams were immediately sent to carry out on site rescue. According to incomplete statistics, 139,642 medical staff participated in the rescue work and 91,298 worked in the affected area.

Dental professionals in China play a critical role in daily medical services. According to estimated statistics (ratio of health humanpower staff to population size) made by the School of Stomatology, Fourth Military Medical University, in 2002, there were 54,877 dental clinics and 249,906 professionally active dental staff in China. This significant pool of experience and human capital makes dental professionals a valuable part of public health emergency response efforts. Dental professionals are incumbent to providing health care to the population in mass casualty events.

The traditional role of dentistry in mass disasters is primarily forensic odontology. ${ }^{3-5}$ However, it is clear that dentists, with their extensive academic training and practical skills, can make a much greater contribution to the medical response to a natural disaster. ${ }^{6,7}$ They can act as the front-line responders and also mitigate prolonged recovery of the dental care infrastructure and improve the odds of having sustainable systems of dental care in the aftermath of devastation. ${ }^{8,9}$ During the rescue work at the Wenchuan 
earthquake, dentists from all over the country played a well-acknowledged role including voluntary donation of blood and relief supplies, financial aid, participation in the emergency medical response team and providing emergency dental treatment and unique professional dental care services for victims and rescue personnel. On the basis of experiences acquired from the Wenchuan earthquake, we will discuss the role of dental professionals in the aftermath of earthquake at three stages: first aid, settlement and reconstruction. We believe that dentists could make more professional contributions to mass casualty and disaster events in the future through well organised services.

\section{STAGE 1 (FIRST AID)}

Stage 1 covers the first ten days after the earthquake; the major tasks in this stage include search and rescue of the disaster victims and providing the first medical response.

\subsection{Participating in the emergency response team}

The mass casualty response to earthquakes includes the four essential elements of disaster medical response: search and rescue; triage and initial stabilisation; definitive medical care; and evacuation. ${ }^{10}$ Survival is directly related to the length of time from the occurrence of the quake until the time of extrication from under the rubble. The survival rate of earthquake victims rescued in the first 24 hours is about 90\%; 50\%-60\% for the second 24 hours; and 20\%-30\% for the third 24 hours. ${ }^{11}$ The earlier the critically injured victim receives medical care, the better the chances of survival. Hence, it is effective to provide first response assistance to victims and coordinate an early medical response. ${ }^{12}$ Dental professionals, by virtue of their commitment to comprehensive primary health care, have valuable assets to bring to the initial response to a natural disaster when the local traditional medical system is overwhelmed..$^{13}$ In the aftermath of the Wenchuan earthquake, many dental staff participated in the first-front medical response team and performed various emergency response activities, including assistance in

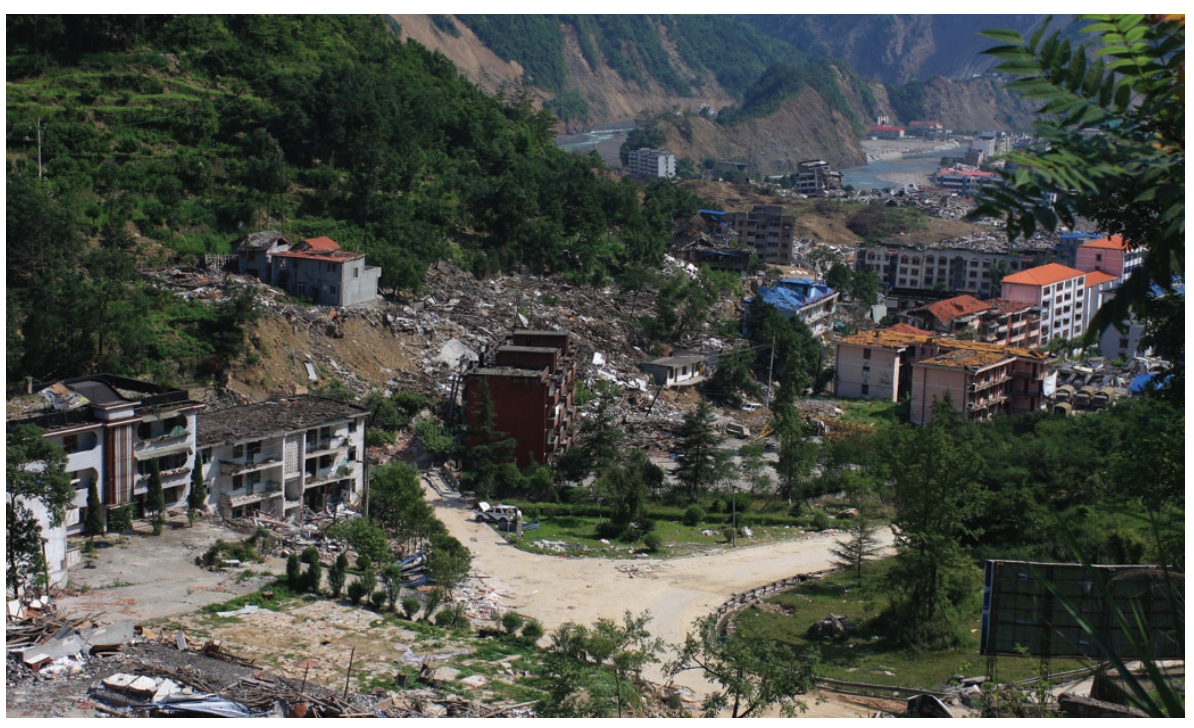

Fig. 1 A general view of the earthquake-hit Beichuan county, which is located in the northeast of the epicentre of Wenchuan county, Sichuan province. The view shows the concrete structures crumbling to rubble under their own weight, or being crushed by landslides

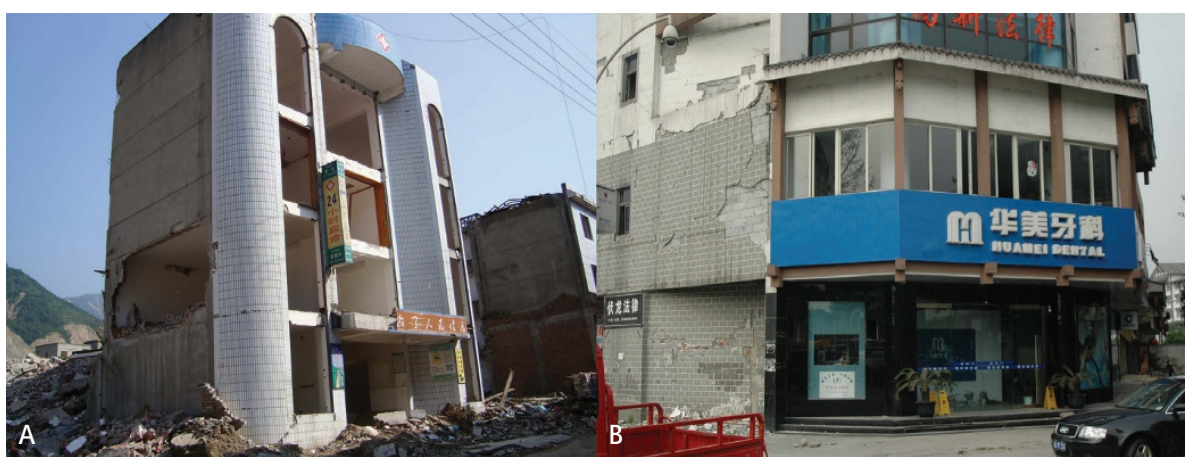

Fig. 2 The view shows the collapsed local hospital $(A)$ and damaged local dental clinic $(B)$ in the earthquake-hit Dujiangyan city

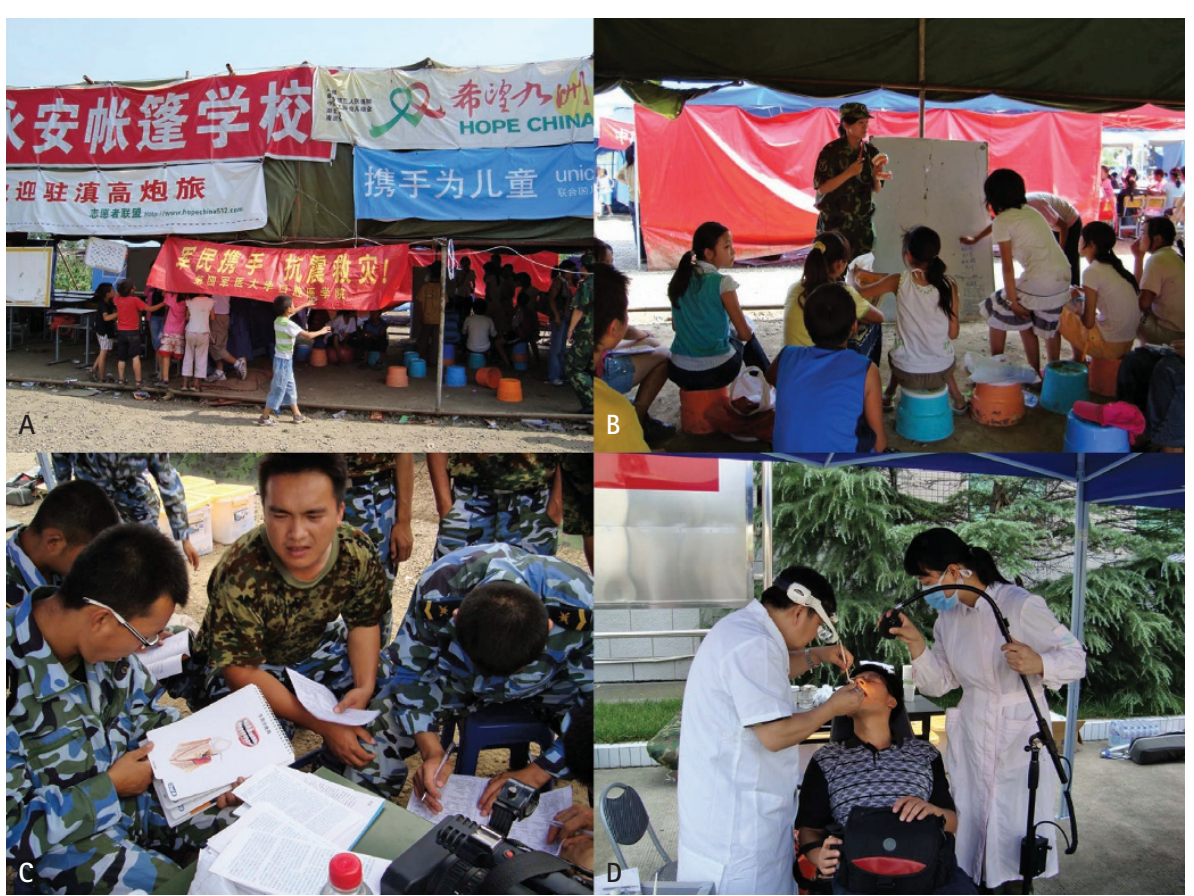

Fig. 3 In the aftermath of Wenchuan earthquake, dental professionals actively provided oral health services for the victims and rescue personnel. A) The tent school; B) The military dentist teaching the children how to keep good oral health habits; C) The rescue personnel reading the oral health education leaflets during rest time; D) The mobile dental equipment used to implement the emergency dental treatment 
fatality management, triage and prehospital care, drugs dispensing, and emergency dental treatment. On 15 May 2008, the military dental officers sent by the school of stomatology, Fourth Military Medical University, undertook dental, oral and maxillofacial surgery for all the victims in Mianyang city and administered anaesthesia for all the surgical operations in the local Changgang Hospital, which greatly enhanced the surge capacity of the local medical system. On 18 May 2008, dentists from the stomatological college, Beijing University participated in evacuating the wounded from Mianyang city to Chongqin Municipal area (400 km away).

\subsection{Sufficient ward beds}

Facing more and more injured victims from the Wenchuan earthquake and with an overwhelming burden to the local hospitals, the stomatological hospitals in the unaffected area went on emergency standby for 24 hours. The emergency rescue area for injured victims from the Wenchuan earthquake was established and green corridors were opened as well. All procedures were simplified and ward beds in the department of oral and maxillofacial surgery were reserved in case of sudden events. On 23 May 2008, the injured victims were transported to the school of stomatology, Fourth Military Medical University and the oral and maxillofacial surgeons successfully treated mandibular fractures. After the initial danger period, victims were transferred to the main hospital for further rehabilitation. Departments of oral and maxillofacial surgery should fully utilise their expertise and make all efforts to provide the best medical therapy in such situations. ${ }^{14}$

\subsection{Donation of oral hygiene products}

The rescue troops and disaster victims lived in the temporary shelter and living conditions were quite poor with severe deficiencies in supplies of food, medicine and tap water. Moreover, the rescue troops and disaster victims were under huge physical and psychological stress. Hence, daily oral cleaning and conventional oral health care maintenance was difficult to carry out. In such an environment it was extremely easy for oral disease to occur.

By means of an investigation, the oral ulcer, periodontal diseases and pericoronitis were detected at different degrees, which seriously affected the health of rescue personnel and victims. ${ }^{2}$ On the morning of 16 May, the dean of the local stomatological hospital collected information in the affected area and urgently requested oral hygiene products from the school of stomatology, Beijing University. On the evening of 16 May, the first 50 cases of 4,000 bottles of rinse solution were distributed to the rescue personnel and the victims in the severely affected cities including Mianzu, Shifang and Dujiangyan. The other 150 cases were sent out on 18 and 19 May in succession.

\section{STAGE 2 (SETTLEMENT STAGE)}

Stage 2 covers day 3 to day 60 after the earthquake, the major task of this stage being to house the disaster population. When local medical systems were able to accommodate the requirements of this community, the role that dental professionals could play changed. On 31 May 2008, we carried on the investigation in the Dujiangyan disaster area and found that six dental clinics had been forced to close down as they were not safe and the local hospitals did not provide oral health services (Fig. 2). There was great demand for dental treatment from the disaster victims in the temporary shelter. Early dental treatment or early initiation of oral disease prophylaxis can have a significant influence on quality of life. Hence, at this stage, dental professionals need to undertake greater responsibility for recovery of oral health care.

\subsection{Providing oral health services}

Under the circumstances, the disaster population could not continue good tooth cleaning. The deposition of food and increased tartar can easily cause gingivitis, pulpitis and periapical periodontitis. In addition, the disaster victims had to withstand the huge psychological stress of the earthquake, accompanying over-fatigue, insufficient sleep and extremely decreased immunity, which possibly caused oral mucous diseases such as oral ulcer and lichen planus.
Furthermore, many victims were injured to different degrees. For example, jaw fracture would lead to limitation of mouth opening, difficulty in diet, talking and oral cleaning. Other bone fractures could cause the casualties to have difficulties moving around which in turn would affect their ability to maintain oral care. The medical infrastructure in the disaster area was seriously damaged; preventive dentistry could not be implemented normally and the first response team was not equipped with dental facilities. Hence, dental professionals should assist in the rapid placement of mobile and portable dental equipment to implement the prevention and treatment of oral diseases such as acute dental caries, dental pulp disease, acute pericoronitis, peridontitis and oral ulcer in the aftermath of disaster. Moreover, they should provide oral health education and counselling. Dental practice could be carried out independently or in conjunction with the local hospital or clinic to serve the relief personnel, victims and local students. It was reported that, up to the end of May, thousands of volunteer troops and about 200,000 volunteers took an active part in victim rescue and treatment. During stage 2, dental volunteers were in great demand to carry on the oral health care for numerous victims in 'tent school' and the temporary shelter.

\subsection{Implementing oral health education}

The huge earthquake not only caused massive casualties instantaneously, but it also had various effects on people's health. It is therefore imperative to instruct the population to make full use of existing resources to carry on selfcare of oral health. Dental professionals are adept at connecting community-level programmes to state-of-the-art public health initiatives. ${ }^{13}$ In the aftermath of the earthquake, dental staff implemented the oral health education system among the victims and distributed educational material as soon as possible. By means of lectures, posters and dental services, the victims were encouraged to keep up good oral health habits (Fig. 3). More importantly, the Ministry of Health broadcast an oral health education programme on television in the aftermath 
of the earthquake, which greatly benefited all the victims and alleviated the pressure on dental practitioners in the quake-hit area.

\section{STAGE 3 \\ (RECONSTRUCTION STAGE)}

Stage 3 covers day 30 to two years after the earthquake; the major task in this stage being to reconstruct the affected area. The official reported stated that $80 \%$ of medical buildings in Dujiangyan were seriously damaged and could not operate normally and the damage in the urban area was even more serious. The earthquake created chaos and led to the unprecedented destruction of the normal functioning of the affected community, including its public health and medical care infrastructure and resources. The lack of clinics and dentists badly impacted the delivery of oral care services. Therefore, each stomatological hospital in the unaffected area had to provide the technical and financial support to mitigate the prolonged recovery of local dental infrastructure.

\subsection{Rebuilding the oral health care system}

For effective response and recovery, public health dentists should develop an earthquake action plan to reconstruct the three-level health service network of villages, towns and counties and cultivate alliances with health units located inside the region and stomatological hospitals outside the affected area. For example, after the Wenchuan earthquake dental professionals worked with local staff to promote and implement programmes such as oral health needs assessment and planning.

\subsection{Donating dental equipment, instruments and materials}

The medical buildings in the disaster area were seriously damaged and the dental facilities could not be operated normally. The dental equipment, materials and instruments were greatly needed in the quake-hit area, which included dental chairs, the light-curing machine, the ultrasonic tooth-cleaner, the dental X-ray machine, the high temperature autoclave and the dental laboratory table. According to the principle of 'one province helps one severely affected county', the citywide or regional consortium of programmes and hospitals were well organised and could assist in a suitable support mechanism to speed up recovery of the oral health service.

\section{SUMMARY}

A survey of Hawaii and New England dental professionals showed that dentists have the basic knowledge and experience and are willing to perform a variety of roles in being prepared for and in response to a bioterrorist event. ${ }^{15,16}$ Mississippi's experience of Hurricane Katrina demonstrated that the American public expects and demands efficient emergency public health and medical services. They suggested that the dental workforce should be engaged in disaster mitigation to improve recovery and response in the aftermath of natural disasters. ${ }^{9}$ In Illinois, the state's Department of Public Health's Division of Oral Health has successfully integrated oral health care professionals into the emergency medical response system. ${ }^{8}$ In the aftermath of Wenchuan earthquake, the Chinese dental professionals participated actively in the first emergency response team, definitive dental treatment, oral health services and education, and the recovery of local oral care infrastructure and resources. Therefore, the role of dentists in mass casualty events cannot be ignored and they can greatly enhance the surge capacity of the local medical system and provide professional oral health care early to improve quality of life. We strongly urge that emergency preparation planning should be incorporated into modern dental curricula so that dental students and professionals and prepared and trained to respond effectively in mass casualty events. ${ }^{17-19}$ Furthermore, more studies should be carried out to determine how the immediate response to mass casualty events can be improved and how dentistry's role can be enhanced.

1. Wang S Y, Li Y H, Chi G B et al. Injury-related fatalities in China: an under-recognised public-health problem. Lancet 2008; 372: 1765-1773.

2. Li G. The role the dental profession can play in mass casualty and disaster events. Hua Xi Kou Qiang Yi Xue Za Zhi 2008; 26: 347-351.

3. Vale G L, Noguchi T T. The role of the forensic dentist in mass disasters. Dent Clin North Am 1977; 21: 123-135.

4. Kieser J A, Laing W, Herbison P. Lessons learned from large-scale comparative dental analysis following the South Asian tsunami of 2004. J Forensic Sci 2006; 51: 109-112.

5. Pretty I A, Sweet D. A look at forensic dentistry Part 1: The role of teeth in the determination of human identity. Br Dent J 2001; 190: 359-366.

6. Psoter W J, Park P J, Boylan R J, Morse D E, Glotze $D$ L. National emergency response programs for dental health care professionals. J Am Dent Assoc 2008; 139: 1067-1073.

7. Morlang W M. Dentistry's vital role in disaster preparedness. J Calif Dent Assoc 1996; 24: 63-66.

8. Janssen J A, Lampiris L N. Disaster response in Illinois: the role for dentists and dental hygienists. Dent Clin North Am 2007; 51: 779-784, v.

9. Mosca N G. Engaging the dental workforce in disaster mitigation to improve recovery and response. Dent Clin North Am 2007; 51: 871-878, vii-viii.

10. Schnitzer J J, Briggs S M. Earthquake relief - the US medical response in Bam, Iran. N Eng/ J Med 2004; 350: 1174-1176.

11. Wang Z G, Song S M. Medical aid in earthquake disasters. Chin J Traumato/2008; 11: 195-197.

12. Peleg K, Reuveni H, Stein M. Earthquake disasters - lessons to be learned. Isr Med Assoc J 2002; 4: 361-365.

13. Guay A H. The role dentists can play in mass casualty and disaster events. Dent Clin North Am 2007; 51: 767-778, v.

14. Grant W D. Readiness and response: the oral and maxillofacial surgeon's role in disaster. J Oral Maxillofac Surg 2006; 64: 749.

15. Katz A R, Nekorchuk D M, Holck P S et al. Dentists' preparedness for responding to bioterrorism: a survey of Hawaii dentists. J Am Dent Assoc 2006; 137: 461-467.

16. Scott TE, Bansal S, Mascarenhas A K. Willingness of New England dental professionals to provide assistance during a bioterrorism event. Biosecur Bioterror 2008; 6: 253-260.

17. Colvard M D, Lampiris L N, Cordell G A et al. The dental emergency responder: expanding the scope of dental practice. JAm DentAssoc 2006; 137: 468-473.

18. Glotzer D L, Rekow E D, More F G, Godder B, Psoter W. All hazards training: incorporating a catastrophe preparedness mindset into the dental school curriculum and professional practice. Dent Clin North Am 2007; 51: 805-818, vi.

19. Psoter W J, Herman N G, More F G et al. Proposed educational objectives for hospital-based dentists during catastrophic events and disaster response. J Dent Educ 2006; 70: 835-843. 